\title{
An Experimental Study of the Coupling Between the Order-Disorder Transition and the Martensitic Transformation in Cu-Al-Be Shape Memory Alloys
}

\author{
M. Jurado, L. Mañosa, A. Planes and C. Stassis* \\ Departament E.C.M., Facultat de Física, Diagonal 647, E-08028 Barcelona, Catalonia, Spain \\ * Ames Laboratory, U.S. DOE and Department of Physics and Astronomy Iowa State University, Ames, \\ IA 50011 , U.S.A.
}

\begin{abstract}
Order-disorder transitions in $\mathrm{Cu}-\mathrm{Al}-\mathrm{Be}$ alloys have been studied by means of $\mathrm{X}$ ray diffraction measurements. Assuming that different ordered degrees can be "frozen" with suitable heat treatments, their effect on the martensitic transformation has been analized by microcalorimetry. The results indicate a change in the temperature of the martensitic transformation directly related to the change of the order degree: disordering stabilizes the martensite phase. Other effects observed after specific heat treatments have been associated to the vacancies retained after the quench. Additional elastic neutron scattering experiments provide data to fit the temperature changes to a previous proposed model.
\end{abstract}

\section{INTRODUCTION}

A number of $\mathrm{Cu}$-based alloys undergo at a temperature $M_{s}$ a martensitic transformation (MT) from a high-temperature bcc phase to a close-packed structure [1]. Although the close-packed structure is energetically more favorable, the bcc high-temperature phase is stabilized by a large vibrational entropy [2]. The interest of these alloys stems from their technologically important shape-memory properties, which are directly associated to the MT.

Furthermore, most of these alloys undergoing a MT exhibit ordered structures below a certain temperature $T_{c}\left(T_{c}>M_{s}\right)$ [3]: In the case of Cu- $\mathrm{Zn}-\mathrm{Al}$ alloys, it has been shown that the degree of atomic order at low temperature can be controlled by means of suitable heat treatments [4], and that the induced changes affect the relative stability between the bcc and the close-packed phases [5].

$\mathrm{Cu}$-Al-Be alloys display at high temperatures (above 970K), a bcc structure ( $\beta$-phase). Below the range of stability of the $\beta$-phase, the equilibrium phases are the so-called $\alpha$ (fcc structure) and $\gamma_{2}$ $\left(C u_{9} A l_{4}\right.$ stucture) phases [6]. The $\beta$-phase can be metastabilized at lower temperatures by suitable cooling. It is during this cooling that the $\beta$-phase goes through different ordered states. Once metastabilized, below the eutectic point/line, the $\beta$-phase undergoes, upon further cooling, a MT towards a close-packed structure. This close-packed structure is an orthorombic (18R) structure in the case of $\mathrm{Cu}-\mathrm{Al}-\mathrm{Be}$ alloys close to the stoichiometric $\mathrm{Cu}_{3} \mathrm{Al}$ composition.

The aim of this work is to study the coupling between the order-disorder transition and the martensitic transformation in $\mathrm{Cu}$-Al-Be alloys. For this purpose, we have studied the order-disorder transition in a family of composition related Cu-Al-Be alloys by means of X-ray powder diffraction measurements. Additional elastic neutron scattering experiments were performed for completeness. 
We have also used differential scanning calorimetry to measure the change in the thermodynamic quantities characteristic of the MT after different heat treatments.

This paper is organized as follows. In Section 2 we describe the experimental procedure and give the data we obtained on the X-ray diffraction measurements, the neutron scattering experiments and the calorimetric study. In Section 3, the discussion and conclusions of this work are presented.

\section{EXPERIMENTAL DETAILS AND RESULTS}

Single crystals grown by the modified Bridgman method were used in the measurements. Their atomic composition and characteristic temperatures are given in Table I. $T_{c}$ values are taken from the $\mathrm{X}$-ray diffraction results (see next subsection).

Table I: Atomic composition, $T_{c}$ and $M_{s}$ for the investigated alloys. Uncertainties in $T_{c}$ are $\pm 10 K$, and in $M_{s}, \pm 1 K$. The values with $\left(^{*}\right)$ are taken from [7].

\begin{tabular}{|l|r|r|r|r|r|}
\hline sample & $\mathrm{Cu}$ & $\mathrm{Al}$ & $\mathrm{Be}$ & $T_{c}(K)$ & $M_{s}(K)$ \\
\hline cab56 & 74.08 & 23.13 & 2.79 & 813 & 275 \\
cab21 & 73.97 & 23.06 & 2.97 & 803 & 240 \\
cab51 & 73.73 & 22.72 & 3.55 & 793 & 150 \\
cab90 & 71.77 & 22.73 & 5.50 & 788 & - \\
cab91 & 69.77 & 22.19 & 8.04 & 793 & - \\
cabB & 74.24 & 22.84 & 2.91 & - & $227^{*}$ \\
cabC & 73.98 & 23.06 & 2.97 & - & $228^{*}$ \\
\hline
\end{tabular}

\subsection{X-ray diffraction and neutron scattering measurements.}

We started the study of the order-disorder transitions in $\mathrm{Cu}-\mathrm{Al}-\mathrm{Be}$ from the assumption that the different possible ordered structures for the $\beta$-phase are the $A 2$, the $B 2$, and the $D O_{3}$ (or the equivalent $L 2_{1}$ ) [6]. The $D O_{3}$ and $L 2_{1}$ structures are equivalent in the sense that they give rise to the same Bragg reflections, but they can be distinguished by the corresponding intensity values. The Bragg reflections associated to the different ordered structures are: $k, k, 1$ all odd for the $D O_{3}$ or $L 2_{1}$ superstructure (example (111)); $\mathrm{h}, \mathrm{k}, \mathrm{l}$ all even and $h+k+l=4 n+2$ with $\mathrm{n}$ integer for the B2 superstructure (example (200)); h,k,l all even and $h+k+l=4 n$ for the A2 fundamental reflections. For a detailed description of these structures, see [3]. Depending on the different degrees of order within the sublattices the intensity of the associated peaks range from zero to the maximum value.

$\mathrm{X}$-ray powder diffraction measurements were performed in a Bragg-Brentano diffractometer equipped with a high temperature chamber and a position sensitive detector. We used the $\mathrm{Cu} K \alpha$ radiation $(\lambda=1.5418 \AA)$. Pow der specimens were made from the single crystals by using a diamond file. The typical mass of the powder samples was $10^{-2} \mathrm{~g}$. All the measurements were performed in a primaryvaccuum atmosphere in order to avoid oxidation of the powder particles. Due to their small size, surface oxidation might reduce the intensity of $\beta$-phase peaks.

The procedure to study the order-disorder transitions in our system is as follows: First of all, the powder is annealed in the high temperature chamber at a $T_{h}$ temperature in the $\beta$-phase equilibrium region for around 20 minutes; then it is cooled down to room temperature. This cooling takes approximately 4 minutes. This heat treatment is needed to improve the crystallinity of the powder. Measurements start by heating up again the powder to $T_{h}$ and running a large angle range scan ( $>40$ degrees). After this scan, different scans are conducted at selected temperatures: some of them in a small angular region comprising the (111) and (200) peaks, and others around the (220) peak; each scan takes approximately 3 minutes time.

After each measurement at a selected temperature, and prior to a new change in temperature, the powder was heated up again to $T_{h}$ in order to get a fixed initial reference state (A2) for the next measurement, and also to eliminate possible precipitates of the $\alpha$ and $\gamma_{2}$ phases that could have 
grown during the preceding measurement. Each time that the sample was at $T_{h}$, a long scan was performed. Cooling and heating rates were choosen as fast as possible $(2 \mathrm{~K} / \mathrm{s})$ in order to minimize the growth of precipitates.

The procedure explained above was followed for different temperatures and for the first five compositions given in table $1 . T_{h}$ values were $973 \mathrm{~K}$ for cab21 and cab51, 1073K for cab56 and cab90, and 1123 for cab91. The reason for having different $T_{h}$ values is that for some compositions, the peaks of precipitates did not disappear at $973 \mathrm{~K}$ and therefore, a higher $\mathcal{T}_{h}$ value was needed.

The (111), (200) and (220) $\beta$-phase peaks were integrated for each temperature $T$, once corrected for the background. Also, noise and contamination contributions to the peaks were eliminated by substracting, from the integrated area at $\mathrm{T}$, the corresponding integrated area in the previous $T_{h}$ scan. The resulting values are plotted in figure 1 . The integrated intensities of the superlattice peaks have been normalized to the total amount of $\beta$-phase present, by dividing by the (220) intensity. Typical estimated errors are $\sigma \approx 10^{-3}$. The $(220) \beta$-phase peak is at the same scattering angle value as one of the peaks of the $\gamma_{2}$-phase. For this reason, when $\gamma_{2}$-phase has been detected, the integrated area of the (220) $\beta$-phase peak has been corrected for the $\gamma_{2}$-phase contribution.

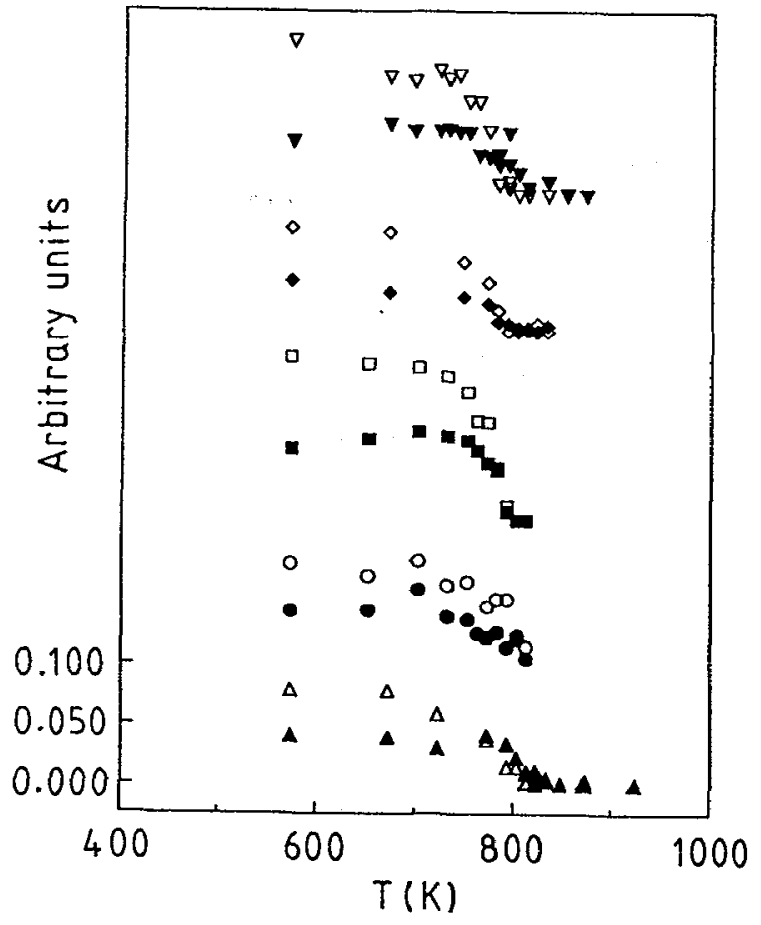

Fig.1. Normalized intensities of superstructure peaks: $\mathrm{I}(111) / \mathrm{I}(220)$ (open symbols), and $\mathrm{I}(200) / \mathrm{I}(220)$ (filled symbols) for cab56 $(\Delta)$, cab21 (o), cab51 ( $(\square)$, cab90 ( () and cab91 ( $)$ compositions.

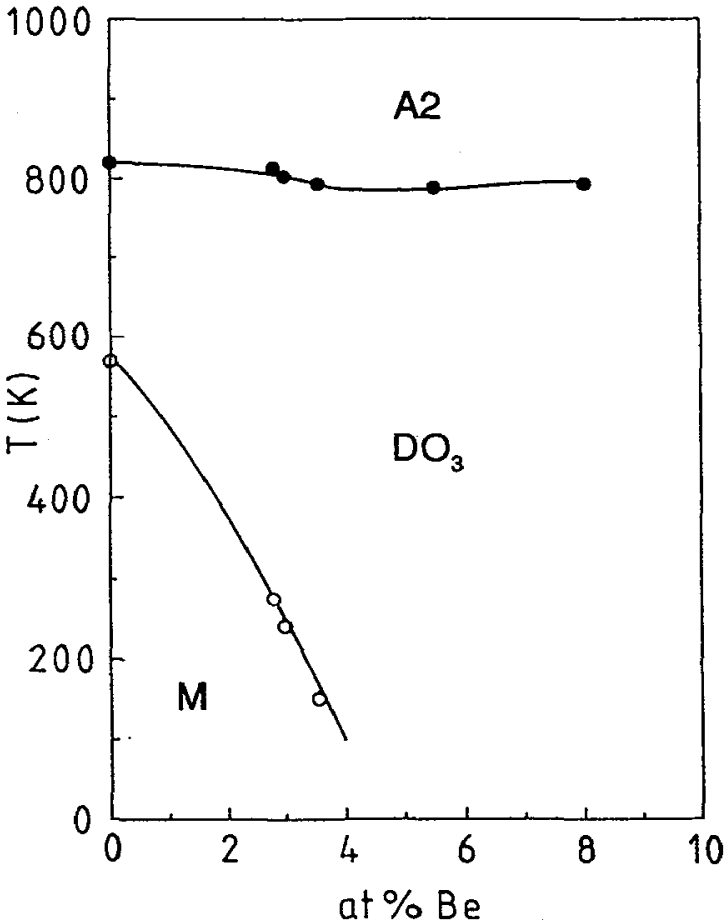

Fig.2. Phase diagram of the $\mathrm{Cu}-\mathrm{Al}-\mathrm{Be}$ investigated alloys: $(\bullet)$ are the $T_{c}$ data and (0) the $M_{s}$ values.

Solid lines are a guide to the eyes.

The (111) and (200) $\beta$-phase peaks were found to appear and grow simultaneously at the same temperature $T_{c}$, which depended on the composition. The fact that the (111) and (200) $\beta$-phase peaks appear together in these $\mathrm{Cu}-\mathrm{Al}-\mathrm{Be}$ alloys indicates that there is a single order-disorder phase transition: from a $A 2$ structure to a $D O_{3}$ (or $L 2_{1}$ ). This order-disorder transition starts at a temperature 
denoted by $T_{c}$. The values found for $T_{c}$ are listed in table I.

Also, the change in the lattice parameter with respect to the value at $T_{h}$ has been determined for the $(220)$ reflection. In addition to the normal variation with temperature, a small discontinuity in the lattice parameter has been observed at a temperature very close to the $T_{c}$ value.

We have computed the different structure factors assuming a uniform mean thermal vibrations in all the sublattices. The values found for (111) and (200) intensity are in better agreement with a $\mathrm{DO}_{3}$ superstructure than with a $L 2_{1}$.

In figure 2, we present the phase diagram obtained from the X-ray measurements and from the calorimetric results (see next subsection). The $M_{s}$ and $T_{c}$ values for the stoichiometric $C u_{3} A l$ obtained from the literature [8] are also shown. cab90 and cab91 samples do not transform into martensite at any temperature below $77 \mathrm{~K}[9]$. The $T_{c}$ points separate the region of stability of the $A 2$ ordered $\beta$-phase from that of stability of the $D O_{3}$ ordered $\beta$-phase. The $M_{s}$ points separate the region of stability of $\beta$-phase from the region of stability of martensite (M).

Elastic neutron scattering experiments were performed using a constant-incident neutron energy triple-axis spectometer (HB1-A, at ORNL). Two single crystals (cabB and cabC in table 1) of $14.72 \mathrm{~g}$ and $14.83 \mathrm{~g}$ were studied prior and after heat treatment. The heat treatments consisted in annealing the sample at $1085 \mathrm{~K}$ for 20 or 30 minutes, air-cooling down to $T_{q}$ and quench into water at room temperature. $T_{q}$ values were $633 \mathrm{~K}, 6.53 \mathrm{~K}$ and $1073 \mathrm{~K}$. It was found that before any heat treatment there was a small arnount of precipitates (mainly $\alpha$ ) and martensite phase in the samples, which did not change appreciably after the different heat treatments. In all cases the amount of precipitates in the sample was extremely small (typically, the relation between maximum intensities was $1 / 1000$ ) in comparison with $\beta$ phase peaks. Nevertheless a striking feature consisting of a nonuniform distribution of the precipitates was found: they showed symmetric distribution along specific crystal directions.

Also, no change in the $T A_{2}$ [110] phonon branch was observed after the heat treatments. Neutron scattering experiments to compute the degree of disorder achieved after the quench have showed that after a heat treatment in sample $C$ with $T_{q}=1073 K$, the $(111) /(220)$ and $(200) /(220)$. values have been reduced to the $10 \%$ and $15 \%$ respectively. The same measurement performed on a $\mathrm{Cu}-\mathrm{Zn}-\mathrm{Al}$ sample, gives changes of the order of $20 \%$.

\subsection{Calorimetric measurements.}

In our calorimetric study we used single crystals cab56, cab21 and cab51 of masses typically between 0.4 and $0.8 \mathrm{~g}$. Scanning calorimetric measurements were made on a high sensitivity microcalorimeter. The technical details are given elsewhere [10].

The single crystals are subjected to different heat treatments: First, the sample is annealed at $T_{a}$ for 3 or 10 minutes. Then, it is air cooled down to a temperature $T_{q}$ and it is quenched into either a mixture of ice and water (cab21, cab51), or water at room temperature (cab56). Just after the quench, a first calorimetric run is performed. 23 hours after the first run, a second calorimetric run was conducted in order to separate non-permanent effects from permanent effects. The $T_{a}$ values used were $973 \mathrm{~K}$ for cab21 and cab51 and $1073 \mathrm{~K}$ for cab51 and cab56. These values were found to be the best suitable for these compositions.

Different heat treatments were conducted on the different specimens for a set of $T_{q}$ values ranging from $350 \mathrm{~K}$ to $1075 \mathrm{~K}$. Also, for all the set of compositions, a reference heat treatment was performed: This reference heat treatment consisted of an air cooling from $T_{a}$ down to room temperature and an annealing of two days prior to the calorimetric runs. The $M_{s}$ values appearing on table 1 are those obtained from these reference heat treatments.

From the recorded thermal curves the martensite start and finish temperatures $\left(M_{s}, M_{f}\right)$ can in principle be obtained. We adopted as a characteristic temperature of the transformation the temperature of the maximum of the thermal curve $\left(T_{M}\right)$. That choice was motivated by the difficulty of a precise determination of $M_{s}$, due to the jerky character of the transition and the presence of 
some premonitory effects, both induced by some of the heat treatments (see [11]) in cab56 and cab21. The jerkier character was found for $T_{q}=823 \mathrm{~K}$ for both cab21 and cab56.

In figure 3 , the shift in the heating $T_{M}$ values with respect to the $T_{M}$ value for the reference heat treatment, are plotted for the different compositions and samples as a function of $T_{q}$, both for the first and second calorimetric runs.

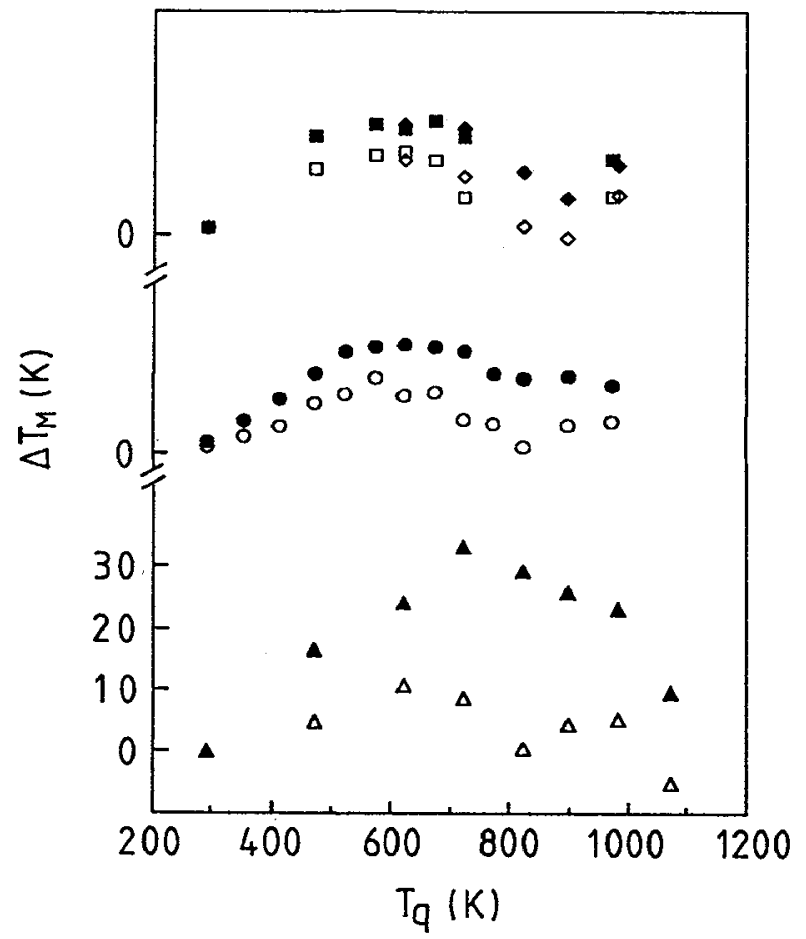

Fig.3. $T_{M}$ minus $T_{M}$ for the reference heat treatment versus $T_{q}$ for the first (filled symbols) and second (open symbols) heating runs. The different composition values have been shifted for a better view: $\operatorname{cab56}(\Delta), \operatorname{cab} 21(0)$ and $\operatorname{cab51(0)},(0)$.

\section{DISCUSSION AND CONCLUSIONS.}

From the X-ray diffraction study it is concluded that in these $\mathrm{Cu}-\mathrm{Al}-\mathrm{Be}$ alloys there is a single order-disorder transition from a $A 2$ structure to a $D O_{3}$ structure (rather than a $L 2_{1}$ ). This transition seems to be a first-order transition according to the change in the lattice parameter at $T_{c}$.

On the other hand, neutron scattering measurements reveal that with the heat treatments one does not increase the amount of precipitates within the samples, but the order degree can be changed. Therefore, one can assume that the heat treatments performed in the calorimetric study modify the order degree in the sample; and that the changes in the $T_{M}$ values are a direct consequence of the coupling between the order-disorder and the martensitic transformations. This is confirmed by the behaviour of the $T_{M}$ values for the second run: They tend to approach the standard heat treatment $T_{M}$ value, wich is considered to be the one with the largest order degree.

In all cases the disorder increases the $T_{M}$ value and, thus, stabilizes the martensite phase. For all the compositions in figure 3 the second run results are qualitatively and quantitatively the same. Also the first run results are similar in the cab51 and cab21. In cab56, an "extra" shift of $T_{M}$ is found in the first heating run (but not in the cooling). We attribute that to a possible stabilization of martensite after the heat treatment for the $M_{\mathrm{s}}$ value in cab56 is close to the room temperature 
value and it is increased with heat treatments. Just after the quench the cab56 sample is likely to be in martensite phase. In cab56 the maximum difference in $T_{M}$ for the first and second heating run (see fig.3) corresponds to $T_{q}=823 \mathrm{~K}$, so following our hypothesis, this $T_{q}$ value would correspond to the maximum amount of martensite stabilized by the quench. If one assumes that the quenched-in vacancies and vacancy clusters are responsible for the stabilization of martensite [12], the larger amount of retained vacancies is reached for $T_{q}=823 \mathrm{~K}$. Also, the jerkier character of the MT was observed for $T_{q}=823 \mathrm{~K}$, then it seems logical to conclude that the interface pinning caused by vacancies retained after the quench would be the origin of the jerky character of the transformation. In the second runs, the jerkier character was always absent. That can be understood by assuming that the mobility of vacancies in the martensite phase is much lesser than in the $\beta$ phase, similarly to what has been observed by positron annihilation in the $\mathrm{Cu}-\mathrm{Al}-\mathrm{Ni}$ alloy system and in $\mathrm{Cu}-\mathrm{Al}-\mathrm{Be}$ alloys with some $\mathrm{Ti}$ or $\mathrm{Cr}$ impurities [12],[13]. Therefore, once the reverse transformation has taken place, the vacancies can easily be removed and do not produce the jerky effect.

Recently, a theoretical relationship between $T_{M}$ and the order parameter (s) has been proposed [14]. For $T_{q}=1073 K$, neutron scattering results show that $s^{2}\left(T_{q}\right) \sim 0.85 s_{0}^{2}$. Inputing this value in equation (1) of reference [11], we obtain $\delta T_{M}=\delta M_{s} \sim-8 K$. This value is in very good agreement with the change in $T_{M}$ measured calorimetrically for sample B $\left(\delta T_{M}=-10 K\right.$, for $\left.T_{q}=673 K\right)$.

For the cab51 composition, in the X-ray measurements it was noticed that it was easier to avoid the growth of the $\gamma_{2}$ phase in comparison with the other compositions. Also the jerky character of the MT was practically absent in its calorimetric runs. These features could be correlated each other. These points, together with the symmetries showed by the precipitates in the neutron study, indicate that, besides the disorder, other effects such as the precipitates precursors or dislocations play a role in driving the MT. From this work, there is no doubt about the influence of the disorder on the shift of $T_{M}$, but it seems that the disorder has a "specific effect", while the whole MT properties are not solely determined by the degree of order.

Acknowledgements.

CuAlBe samples were kindly provided by S. Belkahla, M. Morin and D. Ríos-Jara (INSA, Lyon). X-ray diffraction experiments were performed at S.C.T. (U.B), Barcelona, with the help of X.Alcover and J.Bassa. Thanks are also due to J. Zarestky (ORNL). We acknowledge CICyT (Spain) for financial support (Project number MAT92-884). M. Jurado acknowledges a fellowship from D.G.R. (Catalonia) and Ames Laboratory for hospitality and financial support. Ames Laboratory is operated for U.S. Department of Energy by Iowa State University under Contract No. W-7405-Eng-82.

\section{References}

[1] H. Warlimont and L. Delaey, Prog. Mater. Sci. 18, 1 (1974).

[2] J. Friedel, J. Phys. Lett. 35, L-35 (1974).

[3] M. Ahlers, Prog. Mater. Sci. 30, 135 (1986).

[4] T. Suzuki, Y. Fujii and A. Nagasawa, Materials Science Forum 56-58, 481 (1990).

[5] A. Planes, R. Romero, M. Ahlers, Acta metall. mater. 38, 757 (1990).

[6] S. Belkahla, PhD. thesis, INSA, Lyon 1990.

[7] A. Planes, Ll. Mañosa, D. Ríos-Jara, and J. Ortín, Phys. Rev. B, 14, 7663 (1992).

[8] P. Maletras, P. Duvall, J. Microsc. Spectrosc. Electron. 4, 623 (1979).

[9] D. Ríos-Jara, private communication.

[10] G. Guénin, J. L. Macqueron, M. Mantel, C. Auguet, E. Cesari, Ll. Mañosa, A. Planes, J. Ortin, C. Picornell, C. Seguí and V. Torra, Proc. ICOMAT' 86, Nara, Japan, p. 794, 1986.

[11] M. Jurado, Ll. Mañosa, and A. Planes, Phys. Rev. B 48, 3540 (1993).

[12] T. Wang, B. Y. Wang, S. H. Zhang, Y. Y. Dong, G. Z. Da, Phys. Stat. Sol. (a), 129, 71 (1992).

[13] Y. Kong, B. Jiang, T. Y. Hsu, B. Wang, and T. Wang, Phys. Stat. Sol. (a), 133, 269 (1992).

[14] A. Planes, E. Vives, and T. Castán, Phys. Rev. B 44, 6715 (1991). 